\title{
Derivations on Trellises
}

\section{Ebadi $\mathbf{D}^{*}$ and Sattari $\mathrm{MH}$}

Department of Pure Mathematics, Azarbaijan Shahid Madani University, Tabriz, Iran

\begin{abstract}
In this paper, we introduce the notion of derivations for a trellis and investigate some related properties of this subject. We give some equivalent conditions under which a derivation is isotone for trellises. Also, we study fixed points and define f-derivation on $T$ and Cartesian derivation on $T_{1} \times T_{2}$.
\end{abstract}

Keywords: Trellis; Derivation; F-derivation; Isotone; Pseudo-order; Associative; Distributive; Fixed point; Ideal; Cartesian; Modular 2000 Mathematics Subject Classification: Primary 06B05, 06B35, 06B99

\section{Introduction}

In 1971, Skala introduced the notions of pseudo-ordered sets and trellises. Trellises are generalization of lattices by considering sets with a reflexive and antisymmetric, but not necessarily transitive. They are also an extension of lattices by postulating the existence of least upper bounds and greatest lower bounds on pseudo-ordered sets similarly as for partially ordered sets [1]. Any reflexive and antisymmetric binary relation $\unlhd$ on a nonempty set $\mathrm{T}$ is called a pseudo - ordered on $\mathrm{T}$ and $(T, \unlhd)$ is called a pseudo - order set or posset [2-4]. Clearly, each partial order is a pseudo-order. A natural example of a pseudo-order on the set of real numbers is obtained be setting $\mathrm{x} \unlhd \mathrm{y}$ if and only if $0 \mathrm{y} \unlhd \mathrm{xa}$ for a fixed positive number a. Two elements $x, y$ are comparable if $x \unlhd y$ or $\mathrm{y} \unlhd \mathrm{x}$. For a subset $\mathrm{L}$ of $\mathrm{T}$, the notions of a lower bound, and upper bound, the greatest lower bound (g.l.b), the least upper bound (l.u.b) are defined analogously to the corresponding notions in a posets [5-8].

Generally the notion of a derivation introduced in algebraic systems such as rings, near-rings, specially in lattice theory. Some properties of a derivation such as isotones of a derivation, the set of fixed points of a derivation and relation of derivations with meettranslation as studied in derivations on trellises already defined by Rai and Bhatta [2] with extra conditions that is made derivations isotone. Many authors investigate other properties of derivations on trellises and other algebraic systems [1-3,5]. Here we introduced the notion of a derivation on trellises with weak conditions. The remainder of this paper is organized as follows. We review the definitions and important theorems of the trellis. An equivalent condition is given for a trellis in terms of isotone derivation. Also, we study fixed points and define $\mathrm{f}$-derivation on $\mathrm{T}$ and Cartesian derivation on $\mathrm{T}_{1} \times \mathrm{T}_{2}$.

\section{Preliminaries}

\section{Definition}

Let $\mathrm{T}$ be a nonempty set. A trellis is a psoset $\langle\mathrm{T}, \unlhd\rangle$ where any two of whose elements have a (g.l.b) and a (l.u.b). Any psoset can be regarded as a diagram(possibly infinite) in which for any pair of distinct points $\mathrm{u}$ and $\mathrm{v}$ either there is no directed line between $\mathrm{u}$ and $\mathrm{v}$, or if there is a directed line from $\mathrm{u}$ to $\mathrm{v}$, there is no directed line from $\mathrm{v}$ to $\mathrm{u}$.

\section{Definition} $\in \mathrm{T}$;

A trellis $\mathrm{T}$ is associative if the following conditions hold for all $\mathrm{x}, \mathrm{y}, \mathrm{z}$

$(x \wedge y) \wedge z=x \wedge(y \wedge z)$ or $(x \vee y) \vee z=x \vee(y \vee z)$.

\section{Example 1.1}

The psoset $\mathrm{A}=\{0, \mathrm{a}, \mathrm{b}, \mathrm{c}, 1\}$ with $0 \unlhd a \unlhd b \unlhd c \unlhd 1,0 \unlhd x \unlhd 1$ for every $\mathrm{x}$ $\in\{\mathrm{a}, \mathrm{b}, \mathrm{c}\}$ and $0 \unlhd 1$ while $\mathrm{a}$ and $\mathrm{c}$ are non-comparable. Then $\mathrm{A}$ is a trellis.

\section{Example 1.2}

Let $\mathrm{A}$ be a set $\{0,1, \mathrm{a}, \mathrm{b}, \mathrm{c}, \mathrm{d}\}$ with the following pseudo-order: $a \unlhd c \unlhd d, b \unlhd d, b \unlhd c \unlhd d, 0 \unlhd x \unlhd 1$ for every $\mathrm{x} \in\{\mathrm{a}, \mathrm{b}, \mathrm{c}, \mathrm{d}\}$ and $0 \unlhd 1$. Then $\mathrm{A}$ is a trellis but not lattice and associative since, $(\mathrm{aVb}) \mathrm{Vd}=\mathrm{d}$ but $\mathrm{a} \vee(\mathrm{b} \vee \mathrm{d})=1$.

Some properties on lattices hold in trellises as following:

$\left.\mathrm{p}_{1}\right) \mathrm{x} \wedge \mathrm{y}=\mathrm{y} \wedge \mathrm{x}, \mathrm{x} \vee \mathrm{y}=\mathrm{y} \vee \mathrm{x}$; (commutatively)

$\left.\mathrm{p}_{2}\right)(\mathrm{x} \wedge \mathrm{y}) \vee \mathrm{x}=\mathrm{x},(\mathrm{x} \vee \mathrm{y}) \wedge \mathrm{x}=\mathrm{x} ;($ absorption $)$

$\left.\mathrm{p}_{3}\right) \mathrm{x} \vee((\mathrm{x} \wedge \mathrm{y}) \vee(\mathrm{x} \wedge \mathrm{z}))=\mathrm{x} \wedge((\mathrm{x} \vee \mathrm{y}) \wedge(\mathrm{x} \vee \mathrm{z}))$.(part-preservation).

Theorem 1.3

Let $(\mathrm{T}, \unlhd)$ be a trellis. Then by taking $\mathrm{x} \wedge \mathrm{y}=\mathrm{g} . \mathrm{l} . \mathrm{b}\{\mathrm{x}, \mathrm{y}\}$ and $\mathrm{x} \vee$ $\mathrm{y}=$ l.u.b $\{\mathrm{x}, \mathrm{y}\}$ the binary operation $\vee$ and $\Lambda$ satisfy in $\mathrm{p}_{1}, \mathrm{p}_{2}, \mathrm{p}_{3}$.

From now on, by trellis $(\mathrm{T}, \mathrm{V}, \wedge)$ we mean $(\mathrm{T}, \unlhd)$ that $\mathrm{x} x \unlhd y$ y is defined by $\mathrm{x} \wedge \mathrm{y}=\mathrm{x}$ or $\mathrm{x} \vee \mathrm{y}=\mathrm{y}$.

\section{Remark 1.4}

It is trivial that every associative trellis is a lattice.

\section{Theorem 1.5}

A set $\mathrm{T}$ with two commutative, absorption and part-preserving operations " $\vee$ ", " $\wedge$ " is a trellis if a $E \mathrm{~b}$ is defined as $\mathrm{a} \wedge \mathrm{b}=\mathrm{a}$ or $\mathrm{a} \vee \mathrm{b}=\mathrm{b}$.

Proof

Refer to [3, page on 219] .

\section{Definition}

(i) A subtrellis $\mathrm{S}$ of a trellis $(\mathrm{T}, \mathrm{V}, \wedge)$ is a nonempty subset of $\mathrm{T}$ such that $\mathrm{a}, \mathrm{b} \in \mathrm{S}$ implise $\mathrm{a} \wedge \mathrm{b}$ and $\mathrm{a} \vee \mathrm{b}$ belong to $\mathrm{S}$.

${ }^{*}$ Corresponding author: Ebadi D, Department of Pure Mathematics, Azarbaijan Shahid Madani University, Tabriz, Iran, Tel: 0989144062802; E-mail: Davod_ebady@yahoo.com

Received July 22, 2017; Accepted December 26, 2017; Published December 31, 2017

Citation: Ebadi D, Sattari MH (2017) Derivations on Trellises. J Appl Computat Math 7: 383. doi: 10.4172/2168-9679.1000383

Copyright: (๑ 2017 Ebadi D, et al. This is an open-access article distributed under the terms of the Creative Commons Attribution License, which permits unrestricted use, distribution, and reproduction in any medium, provided the original author and source are credited. 
(ii) An ideal $\mathrm{I}$ of a trellis $\mathrm{T}$ is a subtrellis of $\mathrm{T}$ such that $\mathrm{i} \in \mathrm{I}$ and $\mathrm{a} \in \mathrm{T}$ imply that a $\wedge \mathrm{i} \in \mathrm{I}$ or equivalently for any $\mathrm{i} \in \mathrm{I}$ and $\mathrm{a} \in \mathrm{T}$, implies $\mathrm{a} \in \mathrm{I}$. Moreover, an ideal $\mathrm{I}$ of a trellis $\mathrm{T}$ is called a prime ideal if $x \wedge y \in T$ implies $x \in T$ or $y \in T$ for all $x, y \in T$. Note that if $I_{1}, I_{2}$ are ideals of a trellis $\mathrm{T}$, so is $\mathrm{I}_{1} \cap \mathrm{I}_{2}$.

A trellis $\mathrm{T}$ is modular if the following condition holds for all $\mathrm{x}, \mathrm{y}, \mathrm{z} \in$ $\mathrm{T} ; x \unlhd z \Rightarrow \mathrm{x} \vee(\mathrm{y} \wedge \mathrm{z})=(\mathrm{x} \vee \mathrm{y}) \wedge \mathrm{z}$

\section{Theorem 1.6}

In any trellis, the following statements are equivalent:

$\unlhd$ is transitive,

The operation $\vee$ and $\wedge$ are associative,

One of the operations $\vee$ or $\wedge$ is associative.

\section{Proof}

Refer to [3, Theorem 3].

As [2, lemma 2.2] by simple argument we have the following lemma:

Lemma 1.7. Let $\mathrm{k}: \mathrm{T} \rightarrow \mathrm{T}$ be a mapping on a trellis $\mathrm{T}$ satisfying the property

$\mathrm{k}(\mathrm{x} \wedge \mathrm{y})=\mathrm{kx} \wedge \mathrm{y}$. Then for all $\mathrm{x}, \mathrm{y} \in \mathrm{T}$ :

i) $x \unlhd y$ implies $k x \unlhd k y$,

ii) $k x \unlhd x$,

iii) $\mathrm{k}(\mathrm{kx})=\mathrm{kx}$, i.e., $\mathrm{k}$ is idempotent,

iv) $\mathrm{k}(\mathrm{x} \wedge \mathrm{y})=\mathrm{kx} \wedge \mathrm{ky}$.

v) the fixed elements of $\mathrm{k}$ ( $\mathrm{x}$ is said to be a fixed element of $\mathrm{k}$ if $\mathrm{kx}=\mathrm{x}$ ) form an ideal of $\mathrm{T}$ which will be called the fixed ideal of $k$, denoted by Fix k,also

Fix $\mathrm{k}=\mathrm{k}(\mathrm{T})$.

\section{Proposition 1.8}

If $A$ is an ideal of a trellis $T$ and $k$ is a mapping $k: T \rightarrow T$ satisfying $k(x$ $\wedge y)=k x \wedge y$, then $k(A)$ is an ideal of $A$ and hence an ideal of $T$.

\section{Proof}

Refer to [2, proposition 2.6]

Although some trellis are not distributive, by a weaker condition we have the following:

\section{Proposition 1.9}

If a trellis $\mathrm{T}$ satisfies the inequality $\mathrm{x} \wedge(\mathrm{y} \vee \mathrm{z}) \mathrm{E}(\mathrm{x} \wedge \mathrm{y}) \vee(\mathrm{x} \wedge \mathrm{z})$, then every mapping $\mathrm{k}$ on $\mathrm{T}$ satisfying $\mathrm{k}(\mathrm{x} \wedge \mathrm{y})=\mathrm{kx} \wedge \mathrm{y}$, implies $\mathrm{k}(\mathrm{x} \vee \mathrm{y})=\mathrm{kx} \vee \mathrm{ky}$.

\section{Proof}

Suppose that $\mathrm{k}$ be a mapping on $\mathrm{T}$ satisfying the property above. For any $\mathrm{x}, \mathrm{y} \in \mathrm{T}$,

$$
\begin{aligned}
& k(x \vee y)=k(x \vee y) \wedge(x \vee y) \\
& \unlhd(k(x \vee y) \wedge x) \vee(k(x \vee y) \wedge y) \\
& =k((x \vee k y \cdot y) \wedge x) \vee k((x \vee y) \wedge y) . \\
& =k x \vee k y
\end{aligned}
$$

Since $\quad x, y \unlhd x \vee y \quad$ we have $\quad k x, \mathrm{ky} \unlhd k(x \vee y) \quad$ and $\quad$ so $k x \vee \mathrm{ky} \unlhd k(x \vee y)$.
Therefore $k(x \vee y) \triangleleft=k x \vee k y$.

\section{On Derivations of Trellises}

\section{Definition}

Let $\langle\mathrm{T}, \wedge \mathrm{T}, \mathrm{V}\rangle$ be a trellis. A mapping $\mathrm{d}$ of a trellis $\mathrm{T}$ into itself is called a derivation of $\mathrm{T}$ if it satisfies the following condition for all $\mathrm{x}, \mathrm{y}$ $\in \mathrm{T}$ :

$$
\mathrm{d}(\mathrm{x} \wedge \mathrm{y})=(\mathrm{d}(\mathrm{x}) \wedge \mathrm{y}) \vee(\mathrm{x} \wedge \mathrm{d}(\mathrm{y}))
$$

We can often $\mathrm{d}(\mathrm{x})$ written as an abbreviation $\mathrm{dx}$.

\section{Example 2.1.}

(i) Let $\mathrm{T}$ be a trellis with the least element 0 . We define a function $d$ by $d x=0$ for all $x \in T$. Then $d$ is a derivation on $T$, which is called the zero derivation.

(ii) Let $d$ be the identity function on a trellis $\mathrm{T}$. Then $\mathrm{d}$ is a derivation on $\mathrm{T}$, which is called the identity derivation.

\section{Example 2.2}

Let $\mathrm{T}=\{0,1, \mathrm{a}, \mathrm{b}\}$ be a trellis with the following pseudo-order: $a \unlhd b, 0 \unlhd x \unlhd 1$ for every $\mathrm{x} \in\{\mathrm{a}, \mathrm{b}\}$ and $0 \unlhd 1$. We define two functions $\mathrm{d}_{1}, \mathrm{~d}_{2}$

$$
d_{1} x=\left\{\begin{array}{ll}
x, & x=0,1 \\
b & x=a \\
a & x=b
\end{array} \quad d_{2} x= \begin{cases}0, & x=0,1 \\
a & x=a \\
a & x=b\end{cases}\right.
$$

$\mathrm{d}_{2}$ is a derivation on $\mathrm{T}$ but $\mathrm{d} 1$ is not a derivation, because $\mathrm{d}_{1}(\mathrm{a} \wedge \mathrm{a})=\left(\mathrm{d}_{1} \mathrm{a} \wedge \mathrm{a}\right)$ implies $b \neq a$.

\section{Example 2.3.}

Let $\mathrm{T}=\{0,1, \mathrm{a}, \mathrm{b}, \mathrm{c}, \mathrm{d}\}$ be a trellis with the following pseudo-order : $a \unlhd c \unlhd d, b \unlhd d, b \unlhd c \unlhd d, 0 \unlhd x \unlhd 1$ for every $\mathrm{x} \in\{\mathrm{a}, \mathrm{b}, \mathrm{c}, \mathrm{d}\}$ and $0 \unlhd 1$. Define $\mathrm{d}: \mathrm{T} \rightarrow \mathrm{T}$ by:

$$
d x= \begin{cases}0, & x=0,1 \\ a & x=a \\ b & x=b, c, d\end{cases}
$$

$\mathrm{d}$ is a derivation on $\mathrm{T}$.

\section{Example 2.4.}

Every mapping $\mathrm{k}$ on trellis $\mathrm{T}$ satisfying $\mathrm{k}(\mathrm{x} \wedge \mathrm{y})=\mathrm{kx} \wedge \mathrm{y}$ is a derivation on $\mathrm{T}$. The above example is a derivation on $\mathrm{T}$ that it does not satisfy in this property.

\section{Remark 2.5}

It should be noted that principle derivation on lattices [7] is not a derivation on a trellis. Because it does not have associative property, necessarily.

\section{Proposition 2.6}

Let $\mathrm{T}$ be a trellis and $\mathrm{d}$ be a derivation on $\mathrm{T}$. Then the following statements hold:

$\mathrm{dx} \unlhd x$

If $\mathrm{I}$ is a ideal of $\mathrm{T}$, then $\mathrm{dI} \subseteq \mathrm{I}$

If $\mathrm{T}$ has a greatest element 1 and $\mathrm{d}$ is a derivation on $\mathrm{T}$, then $\mathrm{dx}=(\mathrm{x}$ $\wedge \mathrm{d} 1) \vee \mathrm{dx}$ for all $\mathrm{x} \in \mathrm{T}$. 
iv) If $\mathrm{T}$ has a least element 0 and a greatest element 1 , then $\mathrm{d} 0=0$ and $d 1 \wedge x \unlhd d x$.

\section{Proof}

(i) If $x \in T$, then $d x=d(x \wedge x)=(d x \wedge x) \vee(x \wedge d x)=x \wedge d x$.

(ii) If $\mathrm{I}$ is an ideal of $\mathrm{T}$, then for any $\mathrm{x} \in \mathrm{T}, d x \unlhd x$. implies that $\mathrm{dx}$ $\in \mathrm{I}$, thus $\mathrm{dI} \subseteq \mathrm{I}$.

(iii) Note that $d x=d(x \wedge 1)=(d x \wedge 1) \vee(x \wedge d 1)=d x \vee(x \wedge d 1)$

(iv) It is trivial that $\mathrm{d} 0=0$. If $\mathrm{x} \in \mathrm{T}$, we have,

$d x=d(x \wedge 1)=(d x \wedge 1) \vee(x \wedge d 1)$ implies $d x=d x \vee(x \wedge d 1)$

then, $\mathrm{d} 1 \wedge \mathrm{xE} \mathrm{dx}$.

By applying proposition 3.6 (iii), in the cases $d 1 \unlhd x$ and $x \unlhd d 1$ we have the following:

\section{Corollary 2.7}

If $\mathrm{T}$ has a greatest element 1 and $\mathrm{d}$ is a derivation on $\mathrm{T}$, then for all $\mathrm{x} \in \mathrm{T}$ we have

$d 1 \unlhd x$ Implies $d 1 \unlhd d x$

$x \triangleleft d 1$ Implies $\mathrm{dx}=\mathrm{x}$.

\section{Corollary 2.8}

Let $\mathrm{T}$ be a trellis with a greatest element 1 and $\mathrm{d}$ be a derivation on $T$. Then $d 1=1$ if and only if $d$ is the identity derivation.

\section{Remark 2.9}

As derivation on trellises for a derivation $\mathrm{d}$ satisfing the dual formula of $d(x \wedge y)=(d x \wedge y) \vee(x \wedge$ dy),i.e. $d(x \vee y)=(d x \vee y) \wedge(x \vee d y)$, implies that $\mathrm{d}$ is a identity derivation.

\section{Proposition 3.1}

Let $\mathrm{T}$ be a trellis and $\mathrm{d}$ be a derivation on $\mathrm{T}$. Then the following conditions are equivalent:

(i) d is the identity derivation;

(ii) $d(x \vee y)=(d x \vee y) \wedge(x \vee d y)$;

Proof

The implication (i) $\Rightarrow$ (ii) is trivial.

Taking $\mathrm{x}=\mathrm{y}$ with together contraction property of $\mathrm{d}$ implies (ii) $\Rightarrow$ (i).

\section{Definition}

Let $\mathrm{T}$ be a trellis and $\mathrm{d}$ be a derivation on T. If $x \unlhd y$ implies $d x \triangleleft d \mathrm{y}$, we call $\mathrm{d}$ is an isotone derivation.

\section{Example 3.2}

The example of $3.2, \mathrm{~d}_{2}$ is an isotone derivation but in $3.3, \mathrm{~d}$ is not an isotone derivation since, $a \unlhd c$ then $\mathrm{da}=\mathrm{a}, \mathrm{dc}=\mathrm{b}$ that $\mathrm{ab}$.

\section{Proposition 3.3}

Let $\mathrm{T}$ be a trellis with a greatest element 1 and $\mathrm{d}$ be a derivation on $T$. If $d$ is an isotone derivation Then $d x=x \wedge d 1$.

\section{Proof}

Since $\mathrm{d}$ is an isotone, then $d x \unlhd d 1$. Note that $d x \unlhd x$, we can get $d x \unlhd(x \wedge d 1)$, by proposition 3.6(iii), $\mathrm{dx}=\mathrm{dx} \vee(\mathrm{x} \wedge \mathrm{d} 1)=\mathrm{x} \wedge \mathrm{d} 1$.

\section{Remark 3.4}

The above proposition illustrates a condition that makes isotone derivation, principle [7]

\section{Lemma 3.5}

Let $\mathrm{T}$ be a trellis and $\mathrm{d}: \mathrm{T} \rightarrow \mathrm{T}$ be a derivation. Then $\mathrm{d}(\mathrm{dx})=\mathrm{dx}$.

\section{Proof}

We can get, $d x \unlhd(\mathrm{d} x \wedge d x) \vee(\mathrm{d}(\mathrm{d} x) \wedge x)=\mathrm{d}(\mathrm{x} \wedge d x)=\mathrm{d}(\mathrm{dx})$ and also by 3.6, $\mathrm{d}(\mathrm{d} x) \unlhd d x$ thus, $\mathrm{d}(\mathrm{dx})=\mathrm{dx}$.

\section{Theorem 3.6}

Let $T$ be a trellis and $d: T \rightarrow T$ be a derivation satisfying $d(x \vee y)=d x$ $\checkmark$ dy. Then for all $\mathrm{x}, \mathrm{y} \in \mathrm{T}$ :
i) $\mathrm{d}$ is a isotone derivation;
ii) $x \unlhd y$ implies $\mathrm{dx}=\mathrm{x} \wedge \mathrm{dy}$;
iii) $d x \wedge y=d x \wedge d y$;

Proof

(i) Let $x \unlhd y$, then $\mathrm{x} \vee \mathrm{y}=\mathrm{y}$ and so $\mathrm{dx} E(\mathrm{dx} \vee \mathrm{dy})=\mathrm{d}(\mathrm{x} \vee \mathrm{y})=\mathrm{dy}$.

Let $x \unlhd y$. Then by (i), $d x \triangleleft d y$ and $d x \triangleleft x$. Therefore $d x \triangleleft x \wedge d y$. Also $x \wedge d y \unlhd d x$ since, $\mathrm{dx}=\mathrm{d}(\mathrm{x} \wedge \mathrm{y})=(\mathrm{dx} \wedge \mathrm{y}) \vee(\mathrm{x} \wedge \mathrm{dy})$.

By definition of the derivation, we have $d x \triangleleft x \wedge d y$ for all $\mathrm{x}, \mathrm{y}$ $\in$ L. Taking $\mathrm{x}=\mathrm{dx} \wedge \mathrm{y}$ and $\mathrm{y}=\mathrm{dx}$ in (ii), we have $\mathrm{d}(\mathrm{dx} \wedge \mathrm{y})=(\mathrm{dx} \wedge \mathrm{y})$ $\wedge \mathrm{d}(\mathrm{dx})=(\mathrm{dx} \wedge \mathrm{y}) \wedge \mathrm{dx}=\mathrm{dx} \wedge \mathrm{y}$. Thus $(\mathrm{dx} \wedge \mathrm{y}) \vee(\mathrm{dx} \wedge \mathrm{dy})=\mathrm{dx} \wedge \mathrm{y}$ implies $d x \wedge d y \unlhd y$. Since $d x \wedge y \unlhd d x$ thus $d(d x \wedge y) \unlhd d y$. Then by above equality we have $d x \wedge y \unlhd d y$. Also $d x \wedge y \unlhd d x$. Then we can get, $d x \wedge y \unlhd d x \wedge d y$. With attention to above inequalities we have $\mathrm{dx} \wedge$ $\mathrm{y}=\mathrm{dx} \wedge \mathrm{dy}$.

\section{Corollary 3.7}

Let $\mathrm{T}$ be a trellis and $\mathrm{d}: \mathrm{T} \rightarrow \mathrm{T}$ be a derivation satisfying $\mathrm{d}(\mathrm{x} \vee \mathrm{y})=\mathrm{dx}$ $V$ dy. Then for all $x, y \in T, d(x \wedge y)=d x \wedge y$.

\section{Proof}

We have $d(x \wedge y) \unlhd d x \wedge d y=d x \wedge y$, since $\mathrm{d}$ is isotone and if $x \wedge y \triangleleft x$ then $d(x \wedge y) \triangleleft d x \wedge d y$. By definition of the derivation, we can get the inverse relation and $\operatorname{so} d(x \wedge y)=d x \wedge y$ for all $x, y \in L$.

\section{Corollary 3.8}

If a trellis T satisfies the inequality $x \wedge(y \vee z) \unlhd(x \wedge y) \vee(x \wedge z)$ and $\mathrm{d}$ be a derivation on $\mathrm{T}$, Then the following conditions are equivalent:

(i) $d(x \vee y)=d x \vee d y$;

(ii) $d(x \wedge y)=d x \wedge y$.

\section{Corollary 3.9}

Let $\mathrm{T}$ be a trellis and $\mathrm{d}$ be a derivation on $\mathrm{T}$. If $\mathrm{d}(\mathrm{x} \wedge \mathrm{y})=\mathrm{dx} \wedge \mathrm{y}$, then $\mathrm{d}(\mathrm{x} \wedge \mathrm{y})=\mathrm{dx} \wedge \mathrm{dy}$.

\section{Proof} $\wedge \mathrm{dx}$

We have $d(x \wedge y)=d x \wedge y$ thus, $d(d(x \wedge y))=d((y \wedge d x))$ then $d(x \wedge y)=d y$

\section{Corollary 4.1}

Suppose $\mathrm{k}$ be a mapping on a trellis $\mathrm{T}$ satisfying $\mathrm{k}(\mathrm{x} \vee \mathrm{y})=\mathrm{kx} \vee \mathrm{ky}$ Then $\mathrm{k}$ is an isotone derivation if and only if $\mathrm{k}(\mathrm{x} \wedge \mathrm{y})=\mathrm{kx} \wedge \mathrm{y}$. 


\section{Remark 4.2}

This corollary implies that inverse relation 2.7 (i) is established.

\section{Proposition 4.3}

Let $\mathrm{T}$ be a trellis and $\mathrm{d}: \mathrm{T} \rightarrow \mathrm{T}$ be a derivation. If $\mathrm{d}(\mathrm{x} \wedge \mathrm{y})=\mathrm{dx} \wedge \mathrm{dy}$ then $\mathrm{d}$ is an isotone derivation.

\section{Proof}

For all $\mathrm{x}, \mathrm{y}$ in T. If $\mathrm{x} \mathrm{E} \mathrm{y}$, then $d x=d(x \wedge y)=d x \wedge d y \unlhd d y$.

\section{Theorem 4.4}

Let $\mathrm{T}$ be a trellis and $\mathrm{d}$ be a derivation on $\mathrm{T}$. Then the following conditions are equivalent:

i) $\mathrm{d}$ is an isotone derivation;

ii) $d x \vee d y \unlhd d(x \vee y)$; .

\section{Proof}

(i) $\Rightarrow$ (ii). By (i), we have $d x \unlhd d(x \vee y), d y \unlhd d(x \vee y)$, and so $d x \vee d y \unlhd d(x \vee y)$.

(ii) $\Rightarrow$ (i). Assume that (ii) holds. Let $x \unlhd y$.

By (ii), $d x \unlhd(d x \vee d y) \unlhd d(y \vee x)=d y$. Thus $d x \triangleleft d y$.

\section{Remark 4.5}

Despite lattices, on trellises we cannot expect the following statements for an isotone derivation $\mathrm{d}$ :

1) $d(x \wedge y)=d x \wedge d y$

2) $d(x \vee y)=d x \vee d y$.

\section{Remark 4.6}

It is trivial that every distributive trellis is a modular trellis and every distributive trellis is a associative trellis. Note that by [4, page on 224] every associative trellis is a transitive trellis, and so every distributive trellis is a lattice.

\section{Definition}

Let $\mathrm{T}$ be a trellis and $\mathrm{d}$ be a derivation on $\mathrm{T}$. Define $\operatorname{Fix}_{\mathrm{d}}(\mathrm{T})=\{\mathrm{x} \in$ $\mathrm{T} \mid \mathrm{dx}=\mathrm{x}\}$. By the following proposition we can see that $\in \mathrm{E} \in \operatorname{Fix}_{\mathrm{d}}(\mathrm{T})$ is down-closed set, that is, $\mathrm{x} \in \operatorname{Fix}_{\mathrm{d}}(\mathrm{T})$ and $y \unlhd x$ imply $\mathrm{y} \in \operatorname{Fix}_{\mathrm{d}}(\mathrm{L})$. Moreover if $d$ is isotone, $\operatorname{Fix}_{d}(T)$ is an ideal of $T$.

\section{Proposition 4.7}

Let $\mathrm{T}$ be a trellis and $\mathrm{d}$ be a derivation on $\mathrm{T}$. If $\mathrm{y}$ Ex and $\mathrm{dx}=\mathrm{x}$, then $\mathrm{dy}=\mathrm{y}$.

\section{Proof}

Suppose $\mathrm{x}, \mathrm{y}$ are arbitrary elements in $\mathrm{L}, y \triangleleft x$, then $\mathrm{y}=\mathrm{x} \wedge \mathrm{y}$. Thus,

$$
\mathrm{Dy}=\mathrm{d}(\mathrm{x} \wedge \mathrm{y})
$$$$
=(\mathrm{dx} \wedge \mathrm{y}) \vee(\mathrm{x} \wedge \mathrm{dy})
$$$$
=(\mathrm{x} \wedge \mathrm{y}) \vee \mathrm{dy}
$$$$
=\mathrm{yVdy}
$$$$
=\mathrm{y}
$$

\section{Theorem 4.8}

Let $\mathrm{T}$ be a trellis and $\mathrm{d}_{1}$ and $\mathrm{d}_{2}$ be two isotone derivations on $\mathrm{T}$. Then $\mathrm{d}_{1=} \mathrm{d}_{2}$ if and only if $\operatorname{Fix}_{\mathrm{d} 1}(\mathrm{~T})=\operatorname{Fix}_{\mathrm{d} 2}(\mathrm{~T})$.

\section{Proof}

Trivially, $d_{1=} d_{2}$ implies Fix $_{d 1}(T)=F_{i x}(T)$. For the converse, for all $x \in T$ since $d_{1} x \in \operatorname{Fix}_{d_{1}}(T)=\operatorname{Fix}_{d 2}(T)$ we have $d_{2} d_{1} x=d_{1} x$. Similarly $\mathrm{d}_{1} \mathrm{~d}_{2} \mathrm{x}=\mathrm{d}_{2} \mathrm{x}$. On the other hand, isotonness of $\mathrm{d}_{1}$ and $\mathrm{d}_{2}$ implies that $d_{2} d_{1} x \unlhd d_{2} x=d_{1} d_{2}$ and $\mathrm{d}_{2} \mathrm{~d}_{1} \mathrm{x}=\mathrm{d}_{1} \mathrm{~d}_{2} \mathrm{x}$. Also, $\mathrm{d}_{1} \mathrm{~d}_{2} \mathrm{x} E \mathrm{~d}_{2} \mathrm{~d}_{1} \mathrm{x}$, this show that $\mathrm{d}_{2} \mathrm{~d}_{1} \mathrm{x}=\mathrm{d}_{1} \mathrm{~d}_{2} \mathrm{x}$. It follows that $\mathrm{d}_{1} \mathrm{x}=\mathrm{d}_{2} \mathrm{~d}_{1} \mathrm{x}=\mathrm{d}_{1} \mathrm{~d}_{2} \mathrm{x}=\mathrm{d}_{2} \mathrm{x}$.

\section{Definition}

Let $\left(A_{1}, \unlhd_{1}\right)$ and $\left(A_{2}, \unlhd_{2}\right)$ two pseudo-ordered set. By $\left(A_{1} \times A_{2}, \unlhd\right)$ we means the set $\mathrm{A} 1 \times \mathrm{A}_{2}$ with the pseudo-order $\left(a_{1}, a_{2}\right) \unlhd\left(b_{1}, b_{2}\right)$ if and only if $a_{1} \unlhd_{1} b_{1}$ and $a_{2} \unlhd_{2} b_{2}$. If $\mathrm{T}_{1}$ and $\mathrm{T}_{2}$ are trellises, so is $\mathrm{T}_{1} \times \mathrm{T}_{2}$.

\section{Remark 4.9}

$\left\langle\mathrm{T}_{1}, \wedge 1, \mathrm{~V} 1\right\rangle,\left\langle\mathrm{T}_{2}, \wedge 2, \mathrm{~V} 2\right\rangle$ are trellises. It consider that for all $\mathrm{a}_{1}, \mathrm{~b}_{1} \in$ $\mathrm{T}_{1}$ and $\mathrm{a}_{2}, \mathrm{~b}_{2} \in \mathrm{T}_{2},\left(\mathrm{~T}_{1} \times \mathrm{T}_{2}, \mathrm{~V}, \wedge\right)$ with $\left(\mathrm{a}_{1}, \mathrm{a}_{2}\right) \wedge\left(\mathrm{b}_{1}, \mathrm{~b}_{2}\right)=\left(\mathrm{a}_{1} \wedge \mathrm{b}_{1}, \mathrm{a}_{2} \wedge \mathrm{b}_{2}\right)$ and $\left(\mathrm{a}_{1}, \mathrm{a}_{2}\right) \vee\left(\mathrm{b}_{1}, \mathrm{~b}_{2}\right)=\left(\mathrm{a}_{1} \vee \mathrm{b}_{1}, \mathrm{a}_{2} \vee \mathrm{b}_{2}\right)$ is a trellis.

\section{Definition}

Suppose $\mathrm{d}_{1}, \mathrm{~d}_{2}$ are arbitrary derivations on $\mathrm{T}_{1}, \mathrm{~T}_{2}$ respectively. Define $\mathrm{d}: \mathrm{T}_{1} \times \mathrm{T}_{2} \rightarrow \mathrm{T}_{1} \times \mathrm{T}_{2:} \mathrm{d}(\mathrm{a}, \mathrm{b})=\left(\mathrm{d}_{1} \mathrm{a}, \mathrm{d}_{2} \mathrm{~b}\right)$ for all $\mathrm{a} \in \mathrm{T}_{1}, \mathrm{~b} \in \mathrm{T}_{2}$. Trivially, $\mathrm{d}$ is a derivation and it is called a Cartesain derivation.

\section{Example 5.1}

Cartesian derivation of identity derivations is the identity derivation and if $\mathrm{d}_{1}, \mathrm{~d}_{2}$ are isotone derivations then $\mathrm{d}=\mathrm{d}_{1} \times \mathrm{d}_{2}$ is an isotone derivation.

\section{Remark 5.2}

Nonetheless, we can product many derivations in such matter, but there is a derivation on $\mathrm{T}_{1} \times \mathrm{T}_{2}$ that is not a cartesian derivation. For, let

$\mathrm{T}_{1=} \mathrm{T}_{2=} \mathrm{T}=\{0,1\}$ be a trellis with $0 \unlhd 1$ and define $\mathrm{d}$ : $\mathrm{T} \times \mathrm{T} \rightarrow \mathrm{T} \times \mathrm{T}$ by $\mathrm{d}(\mathrm{x}, \mathrm{y})=(\mathrm{x}, \mathrm{y})$ for all $(\mathrm{x}, \mathrm{y})=(1,1)$ and $\mathrm{d}(1,1)=(1,0)$. Note that this derivation is not isotone, perhaps isotone derivation on $T_{1} \times T_{2}$ are Cartesian derivations.

\section{Definition}

Let $\mathrm{T}$ be a trellis. A function $\mathrm{d}: \mathrm{T} \rightarrow \mathrm{T}$ is called an $\mathrm{f}$-derivation on $\mathrm{T}$ if there exists a function $\mathrm{f}: \mathrm{T} \rightarrow \mathrm{T}$ such that

$\mathrm{d}(\mathrm{x} \wedge \mathrm{y})=(\mathrm{d}(\mathrm{x}) \wedge \mathrm{f}(\mathrm{y})) \vee(\mathrm{f}(\mathrm{x}) \wedge \mathrm{d}(\mathrm{y}))$.

for all $\mathrm{x}, \mathrm{y} \in \mathrm{T}$.

\section{Remark 5.3}

It is obvious that if $\mathrm{f}$ is an identity function then $\mathrm{d}$ is a derivation on $\mathrm{T}$.

\section{Example 5.4}

Let $\mathrm{T}=\{0,1, \mathrm{a}, \mathrm{b}, \mathrm{c}\}$ be a trellis with the following pseudo-order: $a \unlhd b \unlhd c, 0 \unlhd x \unlhd 1$ for every $\mathrm{x} \in\{\mathrm{a}, \mathrm{b}, \mathrm{c}\}$ and $0 \unlhd 1$. Define $\mathrm{d}: \mathrm{T} \rightarrow \mathrm{T}$ by:

$$
d x= \begin{cases}0, & x=0, a \\ c & x=b, c \\ a & x=1\end{cases}
$$

Then $\mathrm{d}$ is not a derivation on $\mathrm{T}$ since $0=\mathrm{d}(\mathrm{a} \wedge 1) \neq(\mathrm{da} \wedge 1) \vee(\mathrm{a} \wedge$ $\mathrm{d} 1)=0 \mathrm{~V} \mathrm{a}=\mathrm{a}$. If we define $\mathrm{f}$ by:

$$
f x= \begin{cases}0, & x=0, a \\ c & x=b, c \\ 1 & x=1\end{cases}
$$


then $\mathrm{d}$ is an $\mathrm{f}$-derivation on $\mathrm{T}$, for all $\mathrm{x}, \mathrm{yT}$.

\section{Proposition 5.5}

Let $\mathrm{T}$ be a trellis and $\mathrm{d}$ be a f-derivation on $\mathrm{T}$. Then the following identities hold for all $\mathrm{x}, \mathrm{y} \in \mathrm{T}$

$d x \unlhd f x$

If $\mathrm{T}$ has a least element 0 , then $\mathrm{f} 0=0$ implies $\mathrm{d} 0=0$.

\section{Proof}

(i) For all $\mathrm{x} \in \mathrm{T}$, we have

$\mathrm{dx}=\mathrm{d}(\mathrm{x} \wedge \mathrm{x})=\mathrm{dx} \wedge \mathrm{fx}$, thus $\mathrm{dx} E \mathrm{fx}$.

(ii) Since $d x \unlhd f x$ for all $\mathrm{x} \in \mathrm{T}$, we have $0 \unlhd d 0 \unlhd f 0=0$.

\section{Corollary 5.6}

If $\mathrm{T}$ has a greatest element 1 and $\mathrm{d}$ is an $\mathrm{f}$-derivation on $\mathrm{T}$,

$\mathrm{fl}=1$, then for all $\mathrm{x} \in \mathrm{T}$ we have,

If $f x \unlhd d 1$, then $\mathrm{dx}=\mathrm{fx}$,

If $d 1 \unlhd f x$, then $d 1 \unlhd d x$.

\section{Proof}

(i) we have $d x=d(x \wedge 1)=(d x \wedge f 1) \vee(f x \wedge d 1)=d x \vee f x$, then $f x E d x$. From proposition 3.32 (i), we obtain $\mathrm{dx}=\mathrm{fx}$.

Since $d x=d(x \wedge 1)=(d x \wedge f 1) \vee(f x \wedge d 1)=d x \vee d 1$, we have $d 1 \unlhd d x$.

\section{Remark 5.7}

Note that if $\mathrm{d} 1=1$, since $d 1 \unlhd f 1$, we have $\mathrm{f} 1=1$. In this case from corollary $3.33(\mathrm{i})$, we get $\mathrm{dx}=\mathrm{fx}$.

\section{Definition}

Let $\mathrm{T}$ be a trellis and $\mathrm{d}$ be an $\mathrm{f}$-derivation on $\mathrm{T}$. If $x \unlhd y$ implies $d x \triangleleft d y$, we call $\mathrm{d}$ is an isotone $\mathrm{f}$-derivation .

\section{Example 5.8}

The example of $3.31, \mathrm{~d}$ is not an isotone f-derivation, since $c \triangleleft 1$ but it dose not follow $d c \unlhd d 1$, whereas $\mathrm{f}$ is an increasing function on $\mathrm{T}$.

\section{Corollary 5.9}

Let $\mathrm{T}$ be a trellis and $\mathrm{d}$ be an $\mathrm{f}$-derivation on $\mathrm{T}$. Then for all $\mathrm{x}, \mathrm{y} \in$ T we have,

If $\mathrm{d}$ is an isotone $\mathrm{f}$-derivation, then $d x \vee d y \unlhd d(x \vee y)$.

If $d(x \wedge y)=d x \wedge d y$, then $d$ is an isotone $f$-derivation.

\section{Proof}

(i) We know that $x \unlhd x \vee y$ and $y \unlhd x \vee y$. Since $\mathrm{d}$ is isotone, $\quad d x \unlhd d(x \vee y)$ and $d y \unlhd d(x \vee y)$. Hence we obtain $d x \vee d y \unlhd d(x \vee y)$.

(ii) Let $\mathrm{d}(\mathrm{x} \wedge \mathrm{y})=\mathrm{dx} \wedge \mathrm{dy}$ and $x_{-} y$. Since $\mathrm{dx}=\mathrm{d}(\mathrm{x} \wedge \mathrm{y})=\mathrm{dx} \wedge \mathrm{dy}$, we get $d x \unlhd d y$.

\section{References}

1. Birkhoff G (1967).Lattice theory. Amer Math Soc Providence RI.

2. Rai B, Bhatta SP (2015) Derivations and Translations on Ttrellises. Ann of Math 118: $215-240$.

3. Skala HL (1971) Trellis theory. Algebra Universalis 1: 218-233.

4. Skala H (1972) Trellis theory. American Mathematical Soc 121.

5. Szśz G (1964) Introduction to lattice theory. Academic Press.

6. Szász G (1975) Derivations of lattices. Acta Scientiarum Mathematicarum 37: 149-154.

7. Xin XL, Li TY, Lu JH (2008) On derivations of lattices. Information Sciences 178: 307-316.

8. Yilmaz C, Ozturk MA (2008) On f-derivations of Lattices. Bull Korean Math Soc 4: 701- 707. 